\title{
SYNTHESIS OF 2-TRIFLUOROMETHYL-4,7-DIHYDRO-7- OXO-(1,2,4)TRIAZOLO[1,5-a]PYRIMIDINE-6-CARBOXYLIC ACID DERIVATIVES AS POTENTIAL ANTIMYCO- BACTERIAL AND ANTIMICROBIAL AGENTS
}

\author{
Nawal A. El-Koussi
}

Department of Medicinal Chemistry, Faculty of Pharmacy, Assiut University, Assiut 71526, Egypt

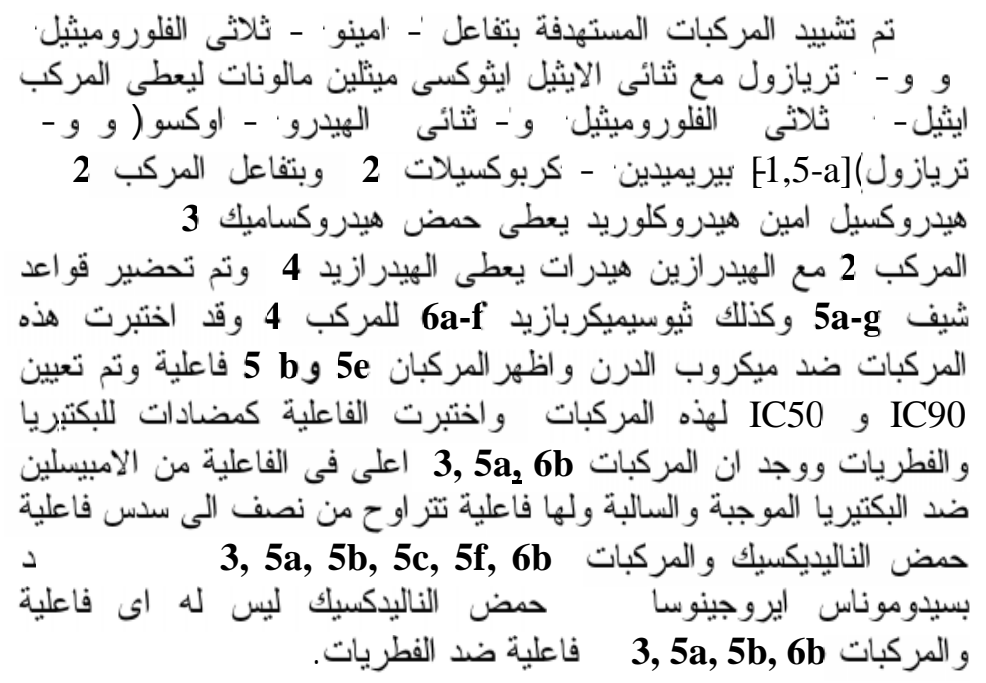

Syntheses of the target compounds were achieved by reaction of 3-amino-5-trifluoromethyl-1,2,4-triazole $\mathbf{1}$ and diethylethoxymethylenemalonate (DEEM) in glacial acetic acid to afford ethyl 2-(trifluoromethyl)-4,7-dihydro-7-oxo[1,2,4]-triazolo[1,5a]pyrimidine-6-carboxylate 2. Reaction of compound $\mathbf{2}$ with hydroxylamine hydrochloride gave hydroxamic acid 3, while reaction with hydrazine hydrate in methanol gave the corresponding carbohydrazide 4. Schiff bases of compound 4 with appropriate aldehyde yielded series $\mathbf{5 a - g}$. Refluxing of hydrazide $\mathbf{4}$ with appropriate isothiocyanate gave thiosemicarbazides $\mathbf{6 a}-f$.

The antimycobacterial evaluation was determined against Mycobacterium tuberculosis $H_{37} R v$ (ATCC 27294). Compound $5 \boldsymbol{e}$ and $\mathbf{5 b}$ showed activity with IC90(6.672, $7.362 \mu \mathrm{g} / \mathrm{ml}$ respectively) 
and IC50 (4.627, $6.382 \mu \mathrm{g} / \mathrm{ml}$ respectively). In vitro antibacterial screening for the prepared compounds were determined against certain strains of gram positive and gram negative bacteria. The results showed that compounds $3, \mathbf{5} \boldsymbol{a}_{\mathbf{2}} \mathbf{6} \boldsymbol{b}$ possessed higher activity than ampicillin against all strains, also the activity range from half to sixth activity of nalidixic acid against E. coli. Compounds 3, 5a, $5 \boldsymbol{b}, \mathbf{5 c}, \mathbf{5 f}, \boldsymbol{6} \boldsymbol{b}$ exhibited activity against $P$. aeruginosa, while nalidixic acid possessed no activity. Compounds $\mathbf{3}, \mathbf{5 a}, \mathbf{5 b}$ and $\mathbf{6 b}$ possessed antifungal activity.

\section{INTRODUCTION}

Fluorine-containing heterocyclic compounds have received considerable interest owing to their potent pharmacological activity $^{1 \& 2}$.

Fluoroquinolones have attracted much attention because of their broad spectrum of activity against various bacteria, mycobacteria, and parasites ${ }^{3}$.

Among the quinolone class of antibacterial agents, fluoroquinolones have shown promise in curing tuberculosis in combination therapy with anti-tuberculosis drugs. These fluoroquinolones are found to be highly concentrated in the host cells, which further enhance their antimycobacterial action. Fluoroquinolones act by interfering with the action of the bacterial DNA gyrase, which results in the degradation of the chromosomal DNA and leads to termination of chromosomal replication and interference with cell division and gene expression ${ }^{4}$. Antibacterial quinolones have attracted increasing attention as clinically useful drugs 5 .

Several analogs in which the fused pyridine nucleus of nalidixic acid was replaced by a five membered ring system as thieno $^{6}$, isothiazolo ${ }^{7}$, furo ${ }^{8}$, pyrolo $^{9-12}$ and triazolo ${ }^{13 \& 14}$ have been reported to have a good antibacterial activity.

A new series of potential antibacterial agents which have a pyridone ring built on a 1,2,4-triazole nucleus was synthesized and evaluated in vitro for antibacterial activity $^{2}$. In this research we used 3amino-5-trifluoromethyl-1,2,4-

triazole for the synthesis of new 2trifluoromethyl-4,7-dihydro-7-oxo$(1,2,4)$ triazolo[1,5-a]pyrimidine-6carboxylic acid derivatives, and evaluated their antimycobacterial and antimicrobial activity.

\section{EXPERIMENTAL}

\section{Materials and Methods}

Melting points were determined on electrothermal melting point apparatus and are uncorrected. Elemental microanalyses were performed on Perkin-Elmer, 240 Elemental Analyzer, at the central laboratory, Assiut University. TLC was carried out using silica gel 60 $\mathrm{F}_{254}$ precoated sheets (E. Merck, 
Germany) and was visualized using UV lamp at $254 \mathrm{~nm}$. IR spectra were recorded as $\mathrm{KBr}$ disks on a 470 Shimadzu IR Spectrophotometer at the Faculty of Pharmacy, Assiut University. ${ }^{1} \mathrm{H}$-NMR spectra were recorded on a JEOL JNM-AL 300 FT NMR system. Chemical shifts were reported in parts per million $(\delta)$ relative to tetramethylsilane (TMS) as internal standard. DMSO- $\mathrm{d}_{6}$ was used as solvent.

\section{Chemistry}

3-Amino-5-trifluoromethyl-1,2,4triazole (1)

was prepared in $92 \%$ yield by reaction of trifluoroacetic acid with aminoguanidine bicarbonate as reported $^{15}$.

\section{2-(Trifluoromethyl)-4,7-dihydro-7-} oxo-1,2,4-triazolo[1,5-a]pyrimidine6-carboxylic acid ethyl ester (2)

To a stirred solution of 3-amino-5trifluoromethyl-1,2,4-triazole $\mathbf{1}(0.373$ $\mathrm{g}, 0.0025 \mathrm{~mol}$ ) in $5 \mathrm{ml}$ glacial acetic acid, diethyl ethoxymethylene malonate (DEEM) (0.86 g, 0.004 mol) was added dropwise. The reaction mixture was refluxed for $3 \mathrm{~h}$ and refrigerated overnight. The precipitated solid was filtered off, washed with ethyl acetate and dried. The crude product was crystallized from acetic acid. Yield $(0.48 \mathrm{~g}, 70 \%)$. mp $280-281^{\circ} \mathrm{C}$. IR (KBr) v 3485, 3135, 1734, 1618, 1578, 1520, 1165, 788, 625, 587. ${ }^{1} \mathrm{H}-\mathrm{NMR}$ (DMSO-d ${ }_{6}$ )

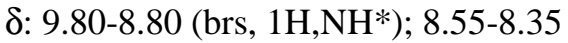

(s, 1H, C-H5); 4.28-3.85 (q, 2H, $\left.\mathrm{CH}_{2} \mathrm{CH}_{3}\right) ; 1.30-0.90 \quad\left(\left(\mathrm{t}, 3 \mathrm{H}, \mathrm{CH}_{2-}\right.\right.$ $\left.\mathrm{CH}_{3}\right)$. Anal. Calcd. for $\mathrm{C}_{9} \mathrm{H}_{7} \mathrm{~F}_{3} \mathrm{~N}_{4} \mathrm{O}_{3}$ : C, 39.1; H, 2.55; N, 20.3; Found C, $39.45 ; \mathrm{H}, 2.4 ; \mathrm{N}, 20.0$ *exchangeable with $\mathrm{D}_{2} \mathrm{O}$

2-(Trifluoromethyl)-4,7-dihydro-7oxo-1,2,4-triazolo[1,5-a]pyrimidine6-hydroxamic acid (3)

To a freshly prepared solution of hydroxylamine [prepared from a solution of hydroxylamine hydrochloride $(7 \mathrm{~g}, \quad 0.1 \mathrm{~mol})$ in methanol $(30 \mathrm{ml})$ by addition while cooling to a solution of potassium hydroxide $(7 \mathrm{~g}, 0.125 \mathrm{~mol})$ in methanol $(30 \mathrm{ml})]$, a solution of compound $2(0.005 \mathrm{~mol})$ in methanol $(20 \mathrm{ml})$ was added portion-wise with stirring at room temperature. After complete addition the mixture was left at room temperature over night. The formed precipitate was filtered off and the potassium salt of hydroxamic acid was dissolved in mixture of water $(15 \mathrm{ml})$, dilute acetic acid $(5 \mathrm{ml})$ and the mixture was allowed to stand for three hours at room temperature. The solid separated was filtered, dried and crystallized from methanol. (Yield $84 \%$, m.p. $\left.226-7^{\circ} \mathrm{C}\right)$. IR(KBr) v3430, 3315, 1647, 1578, 1555.

${ }^{1} \mathrm{H}-\mathrm{NMR}$ (DMSO-d $6, \quad \delta \mathrm{ppm}$ ); 10.72 (s, $1 \mathrm{H}, \mathrm{CONH} *$ ); 8.85-8.60 (bs, $2 \mathrm{H}, \mathrm{NH}^{*}$ of pyrimidine, $\left.\mathrm{OH}^{*}\right) ; 7.13$ (s, 1H, C-H5). *Exchangeable with $\mathrm{D}_{2} \mathrm{O}$ 
2-(Trifluoromethyl)-4,7-dihydro-7oxo-1,2,4-triazolo[1,5-a]pyrimidine6-carbohydrazide (4)

To a stirred ester $2(0.83 \mathrm{~g}, 0.003$ $\mathrm{mol})$ in methanol $(10 \mathrm{ml})$ hydrazine hydrate $(85 \%) 0.43 \mathrm{ml}(0.365 \mathrm{~g}, 0.007$ mol) was added. Stirring was continued at room temperature for 30 minutes and left overnight. The solvent was removed under vacuum, and the residue was crystallized from ethanol. (Yield 75\%, m.p. 200$\left.201^{\circ} \mathrm{C}\right) . \operatorname{IR}(\mathrm{KBr}) \vee 3430,3315,1668$, 1578, 1520, 1165, 788, 625, 587.

${ }^{1} \mathrm{H}-\mathrm{NMR}$ (DMSO-d ${ }_{6}, \delta \mathrm{ppm}$ ); 10.3 (bs, $1 \mathrm{H}, \mathrm{CONH}^{*}$ ), 8.92-8.72 (bs, $2 \mathrm{H}$, $\mathrm{NH}^{*}$ of pyrimidine, C-H5), $4.02-3.58$ (bs, $2 \mathrm{H}, \mathrm{CONHNH}_{2} *$ ) *xchangeable with $\mathrm{D}_{2} \mathrm{O}$.

General method for the synthesis of Schiff bases 5a-g

A solution of $4(0.188 \mathrm{~g}, 0.72$ $\mathrm{mmol})$ in EtOH $(40 \mathrm{ml})$ and the appropriate aldehyde $(0.76 \mathrm{mmol})$ was heated under reflux for $4 \mathrm{~h}$. The precipitate obtained from the hot ethanolic solution or after cooling was purified by crystallization from EtOH. Physical data of the synthesized compounds are shown in Table 1.

\section{N-Benzylidine-2-(trifluoromethyl)-}

4,7-dihydro-7-oxo-1,2,4-triazolo [1,5-a]pyrimidine-6-carbohydrazide (5a)

$\mathrm{IR}(\mathrm{KBr}) \quad v \quad 3440,1713,1627$, 1535, 1508, 1163, 782, 614.

${ }^{1} \mathrm{H}-\mathrm{NMR} \quad\left(\mathrm{DMSO}_{\mathrm{d}}, \quad \delta \mathrm{ppm}\right)$; $12.32\left(\mathrm{~s}, 1 \mathrm{H}, \mathrm{CONH}^{*}\right), 8.82(\mathrm{~s}, 1 \mathrm{H}$, $\mathrm{NH}^{*}$ of pyrimidine), $8.23(\mathrm{~s}, 1 \mathrm{H}, \mathrm{C}-$
H5), 7.95 (s, 1H, N=CH), 7.48-7.37 (m, 5H, ArH). *Exchangeable with $\mathrm{D}_{2} \mathrm{O}$

N-(p-Methoxybenzylidine)-2(trifluoromethyl)-4,7-dihydro-7oxo-1,2,4-triazolo[1,5-a]pyrimidine6-carbohydrazide (5b)

$\mathrm{IR}(\mathrm{KBr}) \quad v \quad 3440,1690,1630$, 1607, 1529, 1504, 1180, 788, 614.

${ }^{1} \mathrm{H}-\mathrm{NMR} \quad\left(\mathrm{DMSO}_{-} \mathrm{d}_{6}, \quad \delta \mathrm{ppm}\right)$; 11.49 (s, $\left.1 \mathrm{H}, \mathrm{CONH}^{*}\right), 8.82(\mathrm{~s}, 1 \mathrm{H}$, $\mathrm{NH}^{*}$ of pyrimidine), 8.34 (s, 1H, C$\mathrm{H} 5), 7.93(\mathrm{~s}, 1 \mathrm{H}-\mathrm{N}=\mathrm{CH}), 7.63-7.57$ (d, 2H, ArH), 6.99-6.96 (d, 2H, ArH), $3.78\left(\mathrm{~s}, 3 \mathrm{H}, \mathrm{OCH}_{3}\right)$. ${ }^{*}$ Exchangeable with $\mathrm{D}_{2} \mathrm{O}$

N-(3,5-Dimethoxybenzylidine)-2(trifluoromethyl)-4,7-dihydro-7oxo-1,2,4-triazolo [1,5-a]pyrimidine-6-carbohydrazide (5c)

$\mathrm{IR}(\mathrm{KBr}) \quad v \quad 3440,1680,1636$, 1605, 1535, 1504, 1180, 775, 635.

${ }^{1} \mathrm{H}-\mathrm{NMR} \quad\left(\mathrm{DMSO}_{6}, \mathrm{~d}_{6}, \delta \mathrm{ppm}\right)$; 12.90 (s, 1H, CONH*), $9.54(\mathrm{~s}, 1 \mathrm{H}$, $\mathrm{NH}^{*}$ of pyrimidine), $7.90(\mathrm{~s}, 2 \mathrm{H}$, $\mathrm{N}=\mathrm{CH}, \mathrm{C}-\mathrm{H} 5), \quad 7.75-7.54 \quad(\mathrm{~m}, 2 \mathrm{H}$, ArH), 7.43-7.40 (m, 1H, ArH), 3.80 $\left(\mathrm{s}, \quad 6 \mathrm{H}, 2\left(\mathrm{OCH}_{3}\right)\right)$. ${ }^{*}$ Exchangeable with $\mathrm{D}_{2} \mathrm{O}$

N-(4-Hydroxy-3-methoxybenzylidine)-2-(trifluoromethyl)-4,7-dihydro-7-oxo-1,2,4-triazolo[1,5-a] pyrimidine-6-carbohydrazide (5d) $\mathrm{IR}(\mathrm{KBr}) \quad \vee \quad 3530,3390,1629$, 1535, 1504, 1165, 788, 614.

${ }^{1} \mathrm{H}$-NMR (DMSO-d $\mathrm{d}_{6}, \delta \mathrm{ppm}$ ); 10.72 (s, $1 \mathrm{H}, \mathrm{CONH}$ ), 9.74 (bs, $1 \mathrm{H}$, $\left.\underline{\mathrm{OH}}^{*}\right), \quad 8.88 \quad\left(\mathrm{~s}, \quad 1 \mathrm{H}, \quad \underline{\mathrm{NH}}^{*} \quad\right.$ of pyrimidine), 8.80 (s, 1H, C-H5), 7.83 
(s, 1H, $\mathrm{N}=\mathrm{CH}), 7.36-7.34(\mathrm{~m}, 1 \mathrm{H}$, ArH), 7.24-7.20 (m, 1H, ArH), 6.986.90 (m, 1H, ArH), 3. 90-3. 80 (s, 3H, $\left.\mathrm{OCH}_{3}\right)$. $*$ Exchangeable with $\mathrm{D}_{2} \mathrm{O}$.

$\mathrm{N}$-( $p$-Dimethylaminobenzylidine)-2(trifluoromethyl)-4,7-dihydro-7oxo-1,2,4-triazolo [1,5-a]pyrimidine-6-carbohydrazide (5e)

$\operatorname{IR}(\mathrm{KBr}) \quad \vee \quad 3525, \quad 3390,1626$ 1515, 1171, 788, 612.

${ }^{1} \mathrm{H}-\mathrm{NMR} \quad($ DMSO-d $6, \quad \delta \quad \mathrm{ppm})$; $11.90(1 \mathrm{H}, \mathrm{CONH} *), 8.88(\mathrm{~s}, 1 \mathrm{H}$, $\mathrm{NH}^{*}$ of pyrimidine), 8.67 (s, $1 \mathrm{H}, \mathrm{C}-$ H5), $7.59(\mathrm{~s}, 1 \mathrm{H}, \mathrm{N}=\mathrm{CH}), 7.66-7.53$ (d, 2H, ArH), 6.72-6.63 (d, 2H, ArH), 2.94-2.87 (s, 6H, N( $\left.\left(\mathrm{CH}_{3}\right)_{2}\right)$. *Exchangeable with $\mathrm{D}_{2} \mathrm{O}$.

$\mathrm{N}$-(p-Chlorobenzylidine)-2(trifluoromethyl)-4,7-dihydro-7oxo-1,2,4-triazolo[1,5-a]pyrimidine6-carbohydrazide (5f)

$\mathrm{IR}(\mathrm{KBr}) \quad v \quad 3440,3390,1627$, 1568, 1536, 1508, 1171, 788, 617.

${ }^{1} \mathrm{H}-\mathrm{NMR} \quad\left(\mathrm{DMSO}_{-} \mathrm{d}_{6}, \quad \delta \mathrm{ppm}\right)$; 11.69 (s, 1H, $\left.\mathrm{CONH}^{*}\right), 8.88(\mathrm{~s}, 1 \mathrm{H}$, $\mathrm{NH}^{*}$ of pyrimidine), 8.80 (s, $1 \mathrm{H}, \mathrm{C}-$ H5), $7.97(\mathrm{~s}, 1 \mathrm{H}, \mathrm{N}=\mathrm{CH}), 7.71-7.65$ (d, 2H, ArH), 7.58-7.47 (d, 2H, ArH). *Exchangeable with $\mathrm{D}_{2} \mathrm{O}$.

\section{N-((Pyridin-3-yl)methylene-2-}

(trifluoromethyl)-4,7-dihydro-7oxo-1,2,4-triazolo [1,5-a]pyrimidine-6-carbohydrazide (5g)

$\mathrm{IR}(\mathrm{KBr})$ v 3440, 3390, 1629, 1565, 1536, 1506, 1171, 785, 612.

${ }^{1} \mathrm{H}-\mathrm{NMR} \quad\left(\right.$ DMSO- $\left.\mathrm{d}_{6}, \quad \delta \quad \mathrm{ppm}\right)$; $12.33\left(\mathrm{~s}, 1 \mathrm{H}, \mathrm{CONH}^{*}\right), 8.92(\mathrm{~s}, 1 \mathrm{H}$, $\mathrm{NH}^{*}$ of pyrimidine), 8.85 (s, $1 \mathrm{H}, \mathrm{C}-$ H5), 8.64-8.61 (m, 2H, pyr), 8.48 (s,
$1 \mathrm{H}, \mathrm{pyr}), 8.24-8.20$ (d, 1H, pyr), 7.58 $(\mathrm{s}, 1 \mathrm{H}, \mathrm{N}=\mathrm{CH})$. ${ }^{*}$ Exchangeable with $\mathrm{D}_{2} \mathrm{O}$.

$\mathrm{N}^{4}$-Substituted-2-(trifluoromethyl)4,7-dihydro-7-oxo-[1,2,4]triazolo [1,5-a]pyrimidine-6-carboxylic acid thiosemicarbazides 6a-f

To a stirred hydrazide 4 (2.62 g, $0.01 \mathrm{~mol})$ in ethanol $(40 \mathrm{ml})$; a solution of the appropriate isothiocyanate $(0.01 \mathrm{~mol})$ in ethanol $(10 \mathrm{ml})$ was added. The mixture was refluxed for two hours. The reaction mixture was cooled and the separated solid was filtered, washed with ethanol and crystallized from appropriate solvent. Physical data are listed in Table 1.

$\mathrm{N}^{4}$ - Methyl 2-(trifluoromethyl)-4,7dihydro-7-oxo[1,2,4]triazolo[1,5-a] pyrimidine-6-carboxylic acid thiosemicarbazide 6a

$\operatorname{IR}(\mathrm{KBr}) \quad v \quad 3435, \quad 3390, \quad 1630$ 1607, 1533, 1502, 1171, 785, 607.

${ }^{1} \mathrm{H}-\mathrm{NMR} \quad\left(\mathrm{DMSO}-\mathrm{d}_{6}, \quad \delta \mathrm{ppm}\right.$ ); 10.85 (br. s, $1 \mathrm{H}, \mathrm{CONH}{ }^{*}$ ); $9.45-9.30$ (br. s, $1 \mathrm{H}, \underline{\mathrm{NH}} * \mathrm{CS}) ; 8.72$ (s, $\mathrm{NH}^{*}$ of pyrimidine), $8.30\left(\mathrm{~m}, 1 \mathrm{H}, \mathrm{NH}^{*} \mathrm{CH}_{3}\right)$; 8.18 (s, 1H, C-H5), 3.30 (s, 3H, $\mathrm{CH}_{3}$ ). *Exchangeable with $\mathrm{D}_{2} \mathrm{O}$.

$\mathrm{N}^{4}$ - Ethyl 2-(trifluoromethyl)-4,7dihydro-7-oxo[1,2,4]triazolo[1,5-a] pyrimidine-6- carboxylic acid thiosemicarbazide $6 \mathrm{~b}$

IR; 3438, 3390,1627, 1607, 1535, 1502, 1175, 785, 612 .

${ }^{1} \mathrm{H}-\mathrm{NMR} \quad\left(\mathrm{DMSO}_{-} \mathrm{d}_{6}, \quad \delta \mathrm{ppm}\right.$ ); 10.90 (br. s, $1 \mathrm{H}, \mathrm{CONH}^{*}$ ); 9.60 (br. s, $\left.1 \mathrm{H}, \underline{\mathrm{NH}^{*}} \mathrm{CS}\right), 8.72-8.65\left(\mathrm{~s}, 1 \mathrm{H}, \mathrm{NH}^{*}\right.$ of pyrimidine $), 8.22 \quad(\mathrm{t}, \quad 1 \mathrm{H}$, 
$\left.\mathrm{NH}^{*} \mathrm{CH}_{2}\right), 7.85$ (s, 1H, C-H5), 3.80$3.70\left(\mathrm{~m}, 2 \mathrm{H}, \underline{\mathrm{CH}_{2}} \mathrm{CH}_{3}\right) ; 1.24-1.15$ (t, $\left.3 \mathrm{H}, \quad \mathrm{CH}_{2} \mathrm{CH}_{3}\right) *$ Exchangeable with $\mathrm{D}_{2} \mathrm{O}$.

Table 1: Physical data of the target compounds.

\begin{tabular}{|c|c|c|c|c|c|c|c|}
\hline $\begin{array}{l}\text { Compd. } \\
\text { No. }\end{array}$ & $\mathrm{R}$ & $\begin{array}{l}\text { M.P } \\
{\left[{ }^{\circ} \mathrm{C}\right]}\end{array}$ & $\begin{array}{c}\text { Yiel } \\
\text { d }\end{array}$ & $\begin{array}{l}\text { Molecular } \\
\text { Formula }\end{array}$ & \multicolumn{3}{|c|}{$\begin{array}{l}\text { Microanalysis } \\
\text { Calcd./Found }\end{array}$} \\
\hline & & & & (M.Wt) & $\mathrm{C}$ & $\mathrm{H}$ & $\mathrm{N}$ \\
\hline 3 & $-\mathrm{NHOH}$ & $\begin{array}{c}226-7 \\
\text { Ethanol }\end{array}$ & 70 & $\begin{array}{c}\mathrm{C}_{7} \mathrm{H}_{4} \mathrm{~F}_{3} \mathrm{~N}_{5} \mathrm{O}_{3} \\
263.13\end{array}$ & $\begin{array}{l}31.95 \\
31.72\end{array}$ & $\begin{array}{l}1.53 \\
1.43\end{array}$ & $\begin{array}{l}6.62 \\
6.41\end{array}$ \\
\hline 4 & $-\mathrm{NHNH}_{2}$ & $\begin{array}{l}200-01 \\
\text { Ethanol }\end{array}$ & 75 & $\begin{array}{c}\mathrm{C}_{7} \mathrm{H}_{5} \mathrm{~F}_{3} \mathrm{~N}_{6} \mathrm{O}_{2} \\
262.15\end{array}$ & $\begin{array}{l}32.07 \\
32.43\end{array}$ & $\begin{array}{l}1.92 \\
2.22\end{array}$ & $\begin{array}{l}2.06 \\
2.48\end{array}$ \\
\hline $5 \mathbf{a}$ & $-\mathrm{NHN}=\mathrm{CH}-\mathrm{C}_{6} \mathrm{H}_{5}$ & $\begin{array}{c}236-7 \\
\text { Ethanol } \\
\end{array}$ & 73 & $\begin{array}{c}\mathrm{C}_{14} \mathrm{H}_{9} \mathrm{~F}_{3} \mathrm{~N}_{6} \mathrm{O}_{2} \\
350.26\end{array}$ & $\begin{array}{l}48.01 \\
48.35 \\
\end{array}$ & $\begin{array}{l}2.59 \\
2.73 \\
\end{array}$ & $\begin{array}{l}3.99 \\
4.18 \\
\end{array}$ \\
\hline $5 b$ & $-\mathrm{NHN}=\mathrm{CH}-p-\mathrm{OCH}_{3} \mathrm{C}_{6} \mathrm{H}_{4}$ & $\begin{array}{c}254-6 \\
\text { Ethanol// } \\
\text { DMF }\end{array}$ & 83 & $\begin{array}{c}\mathrm{C}_{15} \mathrm{H}_{11} \mathrm{~F}_{3} \mathrm{~N}_{6} \mathrm{O}_{3} \\
380.28\end{array}$ & $\begin{array}{l}47.38 \\
47.53\end{array}$ & $\begin{array}{l}2.92 \\
3.21\end{array}$ & $\begin{array}{l}22.10 \\
22.41\end{array}$ \\
\hline $5 c$ & $-\mathrm{NHN}=\mathrm{CH}-3,5-\left(\mathrm{OCH}_{3}\right)_{2} \mathrm{C}_{6} \mathrm{H}_{3}$ & $\begin{array}{c}257-8 \\
\text { Ethanol/ } \\
\text { DMF }\end{array}$ & 98 & $\begin{array}{c}\mathrm{C}_{16} \mathrm{H}_{13} \mathrm{~F}_{3} \mathrm{~N}_{6} \mathrm{O}_{4} \\
410.31\end{array}$ & $\begin{array}{l}46.84 \\
46.75\end{array}$ & $\begin{array}{l}3.19 \\
3.27\end{array}$ & $\begin{array}{l}20.48 \\
20.56\end{array}$ \\
\hline $5 d$ & $\begin{array}{c}-\mathrm{NHN}=\mathrm{CH}-3-\mathrm{OCH}_{3}-4-\mathrm{OH}- \\
\mathrm{C}_{6} \mathrm{H}_{3}\end{array}$ & $\begin{array}{c}239-40 \\
\text { Ethanol/ } \\
\text { DMF }\end{array}$ & 94 & $\begin{array}{c}\mathrm{C}_{15} \mathrm{H}_{11} \mathrm{~F}_{3} \mathrm{~N}_{6} \mathrm{O}_{4} \\
396.28\end{array}$ & $\begin{array}{l}45.46 \\
45.57\end{array}$ & $\begin{array}{l}2.80 \\
2.99\end{array}$ & $\begin{array}{l}21.21 \\
21.54\end{array}$ \\
\hline $5 e$ & $-\mathrm{NHN}=\mathrm{CH}-p-\mathrm{N}\left(\mathrm{CH}_{3}\right)_{2} \mathrm{C}_{6} \mathrm{H}_{4}$ & $\begin{array}{c}265-6 \\
\text { Ethanol/ } \\
\text { DMF }\end{array}$ & 73 & $\begin{array}{c}\mathrm{C}_{16} \mathrm{H}_{14} \mathrm{~F}_{3} \mathrm{~N}_{7} \mathrm{O}_{2} \\
\quad(393.32\end{array}$ & $\begin{array}{l}48.86 \\
48.73\end{array}$ & $\begin{array}{l}3.59 \\
3.42\end{array}$ & $\begin{array}{l}24.93 \\
24.76\end{array}$ \\
\hline $5 f$ & $-\mathrm{NHN}=\mathrm{CH}-p-\mathrm{ClC}_{6} \mathrm{H}_{4}$ & $\begin{array}{c}246-7 \\
\text { Ethanol// } \\
\text { DMF }\end{array}$ & 90 & $\begin{array}{c}\mathrm{C}_{14} \mathrm{H}_{8} \mathrm{ClF}_{3} \mathrm{~N}_{6} \mathrm{O}_{2} \\
384.70\end{array}$ & $\begin{array}{l}43.71 \\
43.52\end{array}$ & $\begin{array}{l}2.10 \\
2.34\end{array}$ & $\begin{array}{l}21.85 \\
21.74\end{array}$ \\
\hline $5 g$ & $-\mathrm{NHN}=\mathrm{CH}-\mathrm{C}_{5} \mathrm{H}_{4} \mathrm{~N}$ & $\begin{array}{c}288-9 \\
\text { Ethanol/ } \\
\text { DMF }\end{array}$ & 65 & $\begin{array}{l}\mathrm{C}_{13} \mathrm{H}_{8} \mathrm{~F}_{3} \mathrm{~N}_{7} \mathrm{O}_{2} \\
351.24\end{array}$ & $\begin{array}{l}44.45 \\
44.82\end{array}$ & $\begin{array}{l}2.30 \\
2.54\end{array}$ & $\begin{array}{l}27.91 \\
27.80\end{array}$ \\
\hline $6 a$ & $-\mathrm{NHNHCSNHCH}_{3}$ & $\begin{array}{c}233-5 \\
\text { Ethanol }\end{array}$ & 72 & $\begin{array}{c}\mathrm{C}_{9} \mathrm{H}_{8} \mathrm{~F}_{3} \mathrm{~N}_{7} \mathrm{O}_{2} \mathrm{~S} \\
335.27\end{array}$ & $\begin{array}{l}32.24 \\
32.51\end{array}$ & $\begin{array}{l}2.41 \\
2.31\end{array}$ & $\begin{array}{l}29.24 \\
29.55\end{array}$ \\
\hline $6 \mathrm{~b}$ & $-\mathrm{NHNHCSNHC}_{2} \mathrm{H}_{5}$ & $\begin{array}{c}221-3 \\
\text { Ethanol }\end{array}$ & 60 & $\begin{array}{c}\mathrm{C}_{10} \mathrm{H}_{10} \mathrm{~F}_{3} \mathrm{~N}_{7} \mathrm{O}_{2} \mathrm{~S} \\
349.29\end{array}$ & $\begin{array}{l}34.39 \\
34.65 \\
\end{array}$ & $\begin{array}{l}2.89 \\
2.64 \\
\end{array}$ & $\begin{array}{l}28.07 \\
28.31\end{array}$ \\
\hline $6 c$ & $-\mathrm{NHNHCSNHC}_{3} \mathrm{H}_{5}$ & $\begin{array}{c}197-8 \\
\text { Ethanol }\end{array}$ & 40 & $\begin{array}{c}\mathrm{C}_{11} \mathrm{H}_{10} \mathrm{~F}_{3} \mathrm{~N}_{7} \mathrm{O}_{2} \mathrm{~S} \\
361.30 \\
\end{array}$ & $\begin{array}{l}36.57 \\
36.74 \\
\end{array}$ & $\begin{array}{l}2.79 \\
2.63 \\
\end{array}$ & $\begin{array}{l}7.14 \\
7.02 \\
\end{array}$ \\
\hline $6 d$ & $-\mathrm{NHNHCSNHC}_{6} \mathrm{H}_{5}$ & $\begin{array}{c}203-4 \\
\text { Ethanol }\end{array}$ & 60 & $\begin{array}{c}\mathrm{C}_{14} \mathrm{H}_{10} \mathrm{~F}_{3} \mathrm{~N}_{7} \mathrm{O}_{2} \mathrm{~S} \\
397.34 \\
\end{array}$ & $\begin{array}{l}42.32 \\
42.68 \\
\end{array}$ & $\begin{array}{l}2.54 \\
2.98 \\
\end{array}$ & $\begin{array}{l}24.68 \\
25.02 \\
\end{array}$ \\
\hline $6 e$ & -NHNHCSNH- $p-\mathrm{CH}_{3} \mathrm{C}_{6} \mathrm{H}_{4}$ & $\begin{array}{c}194-6 \\
\text { Ethanol }\end{array}$ & 60 & $\begin{array}{c}\mathrm{C}_{15} \mathrm{H}_{12} \mathrm{~F}_{3} \mathrm{~N}_{7} \mathrm{O}_{2} \mathrm{~S} \\
411.36\end{array}$ & $\begin{array}{l}43.80 \\
43.56\end{array}$ & $\begin{array}{l}2.94 \\
2.53\end{array}$ & $\begin{array}{l}23.83 \\
24.11\end{array}$ \\
\hline 6f & -NHNHCSNH-c.hexyl & $\begin{array}{c}210-2 \\
\text { Ethanol }\end{array}$ & 50 & $\begin{array}{c}\mathrm{C}_{14} \mathrm{H}_{16} \mathrm{~F}_{3} \mathrm{~N}_{7} \mathrm{O}_{2} \mathrm{~S} \\
403.38\end{array}$ & $\begin{array}{l}41.68 \\
42.03\end{array}$ & $\begin{array}{l}4.00 \\
4.31\end{array}$ & $\begin{array}{l}24.31 \\
24.66\end{array}$ \\
\hline
\end{tabular}


$\mathrm{N}^{4}$ - Allyl 2-(trifluoromethyl)-4,7dihydro-7-oxo[1,2,4]triazolo[1,5-a] pyrimidine-6-carboxylic acid thiosemicarbazide $6 c$

$\mathrm{IR}(\mathrm{KBr}) \quad v \quad 3438, \quad 3395, \quad 1654$, 1631, 1541, 1503, 1181, 785, 613.

${ }^{1} \mathrm{H}-\mathrm{NMR} \quad\left(\mathrm{DMSO}-\mathrm{d}_{6}, \quad \delta \mathrm{ppm}\right)$; 10.51 (s, $\left.1 \mathrm{H}, \mathrm{CONH}^{*}\right), 9.31(\mathrm{~s}, 1 \mathrm{H}$, $\left.\mathrm{NH}^{*} \mathrm{CS}\right), \quad 8.72\left(\mathrm{~s}, \quad \underline{\mathrm{NH}}^{*}\right.$ of pyrimidine), 8.18 (s, 1H, C-H5), 5.79$5.75\left(\mathrm{~m}, 1 \mathrm{H}, \mathrm{CH}=\mathrm{CH}_{2}\right), 5.10-5.01(\mathrm{~m}$, $\left.2 \mathrm{H}, \mathrm{CH}=\mathrm{CH}_{2}\right), 4.10-4.00(\mathrm{~m}, 2 \mathrm{H}$, $\left.\mathrm{NH}-\mathrm{CH}_{2}\right)$. ${ }^{*}$ Exchangeable with $\mathrm{D}_{2} \mathrm{O}$.

$\mathrm{N}^{4}$ - Phenyl- 2-(trifluoromethyl)-4,7dihydro-7-oxo[1,2,4]triazolo[1,5-a] pyrimidine-6- carboxylic acid thiosemicarbazide 6d

$\mathrm{IR}(\mathrm{KBr}) \quad v \quad 3535,3390,1652$, 1629, 1540, 1505, 1185, 784, 612.

${ }^{1} \mathrm{H}$-NMR (DMSO-d ${ }_{6}, \delta \mathrm{ppm}$ ); 10.51 (s, $\left.1 \mathrm{H}, \mathrm{CONH}^{*}\right), 9.78(\mathrm{~s}, 1 \mathrm{H}$, $\left.\underline{\mathrm{NH}^{*}} \mathrm{CS}\right), \quad 8.75 \quad\left(\mathrm{~s}, 1 \mathrm{H}, \underline{\mathrm{NH}}^{*}\right.$ of pyrimidine), 8.74-8.70 (m, $2 \mathrm{H}, \mathrm{NH}^{*}-$ ph, C-H5), 7.27-7.09 (m, 5H, ArH). *Exchangeable with $\mathrm{D}_{2} \mathrm{O}$.

$\mathrm{N}^{4}$ - p-Tolyl -2-(trifluoromethyl)-4,7dihydro-7-oxo[1,2,4] triazolo $[1,5-a]$ pyrimidine-6-carboxylic acid thiosemicarbazide $6 \mathrm{e}$

$\mathrm{IR}(\mathrm{KBr}) \quad v \quad 3435, \quad 3390, \quad 1630$, 1607, 1533, 1502, 1181, 783, 607.

${ }^{1} \mathrm{H}$-NMR (DMSO-d $\left.\mathrm{d}_{6}, \delta \mathrm{ppm}\right)$; 10.50 (s, $\left.1 \mathrm{H}, \mathrm{CONH}^{*}\right), 9.72(\mathrm{~s}, 1 \mathrm{H}$, $\left.\mathrm{NH}^{*} \mathrm{CS}\right), \quad 8.75 \quad\left(\mathrm{~s}, \quad 1 \mathrm{H}, \quad \underline{\mathrm{NH}}^{*}\right.$ of pyrimidine), 8.59 (s, 1H, C-H5), 7.90 (s, 1H, NH*-ph), 7.40-7.37 (d, 2H, ArH), 7.15-7.12 (d, 2H, Ar H), 2.26$2.25\left(\mathrm{~s}, 3 \mathrm{H}, \mathrm{CH}_{3}\right)$ *Exchangeable with $\mathrm{D}_{2} \mathrm{O}$.
$\mathrm{N}^{4}$-Cyclohexyl-2-(trifluoromethyl)4,7-dihydro-7-oxo[1,2,4] triazolo [1,5-a]pyrimidine-6-carboxylic acid thiosemicarbazide $6 \mathrm{f}$

$\mathrm{IR}(\mathrm{KBr}) \quad v \quad 3525 ， 3390,1628$, 1647, 1549, 1525, 1165, 610.

${ }^{1} \mathrm{H}$-NMR (DMSO-d $\left.6, \delta \mathrm{ppm}\right) ;$ 10.50 (br. s, $1 \mathrm{H}, \mathrm{CONH}^{*}$ ); 9.60 (br. s, $\left.1 \mathrm{H}, \mathrm{NH}^{*} \mathrm{CS}\right) ; 8.70\left(\mathrm{~s}, 1 \mathrm{H}, \underline{\mathrm{NH}}^{*}\right.$ of pyrimidine), 8.59 (s, 1H, C-H5), 4.20 (br. s, $\left.1 \mathrm{H}, \mathrm{NH}^{*} \mathrm{C}_{6} \mathrm{H}_{11}\right) ; 2.00-1.00(\mathrm{~m}$, $\left.11 \mathrm{H}, \quad \mathrm{C}_{6} \mathrm{H}_{11}\right) *$ Exchangeable with $\mathrm{D}_{2} \mathrm{O}$.

\section{Antimycobacterial Assay}

The primary antimycobacterial evaluation was performed at the National Hansen`s Disease Programs (NHDP) TAACF facilities, Baton Rouge, LA, USA. The screening was conducted at a single concentration of $6.25 \mu \mathrm{g} / \mathrm{ml}$ against Mycobacterium tuberculosis $\mathrm{H}_{37} \mathrm{Rv}$ (ATCC 27294) in BACTEC 12B medium using the Microplate Alamar Blue Assay (MABA). Compounds exhibiting fluorescence are tested in BACTEC 460-radiometric system $^{16}$. Compounds effecting $<90 \%$ inhibition in the primary screen are not evaluated further.

\section{Antimicrobial assay}

The synthesized compounds were screened in vitro for their antibacterial activity against the following strains Escherichia coli (E. coli, ATCC 25922), Serratia marcescens, DMS 1608, Pseudomonas aeruginosa as gram negative bacteria. Bacillus cereus, and Staphylococcus aureus ATCC 
25923 as Gram positive bacteria. Antifungal activity against Aspergillus niger, Aspergillus flavus, Candida albicans, Scopulariopsis brevicaulis, Fusarium oxysporum and Geotrichum candidum.

\section{Method}

Solutions of the tested compounds, ampicillin trihydrate, nalidixic acid and clotrimazole were dissolved in DMSO and were tested at a concentration $2000 \mu \mathrm{g} / \mathrm{ml}$. The twofold dilution of the compounds and references were prepared. Cultures were grown on nutrient agar medium containing peptone $5 \mathrm{~g} / \mathrm{l}$, beef extract $3 \mathrm{~g} / \mathrm{l}$ and $\mathrm{NaCl} 3 \mathrm{~g} / \mathrm{l}$. The mixture was incubated at $121^{\circ} \mathrm{C}$ and $1.5 \mathrm{~atm}$ for $20 \mathrm{~min}$, distributed in sterile plates $(20 \mathrm{ml}$ per plate) and allowed to solidify. The tested bacterial or fungal species were firstly grown for $48 \mathrm{hr}$, then $1 \mathrm{ml}$ of each suspension was poured on the solidified agar medium and thoroughly distributed on agar surface. Cups were made in the solidified agar (6/plate) with the aid of sterile cork borer. Aliquots of 0.1 $\mathrm{ml}$ of solutions of the tested compounds as well as the reference drugs were pipette into the appropriate cup. The last cup was used as control for the solvent. The plates were left for one $\mathrm{hr}$ at room temperature to attain prediffusion. After $24 \mathrm{hr}$ of incubation at $37^{\circ}$ for bacteria and $48 \mathrm{hr}$ of incubation at $30^{\circ}$ for fungi, the diameters of inhibition zones(mm) were measured and the minimal inhibitory concentrations (MICs) were determined. MICs were recorded as the minimum concentration of a compound that visually inhibits the growth of tested microorganisms. The results are summarized in Table 3 .

\section{RESULTS AND DISCUSSION}

\section{Chemistry}

The synthesis of the target compounds was achieved by the route depicted in Scheme 1. Reaction of 3amino-5-trifluoromethyl-1,2,4-

triazole 1 with diethyl ethoxymethylenemalonate (DEEM) in glacial acetic acid afforded ethyl 2(trifluoromethyl)-4,7-dihydro-7-oxo $[1,2,4]$-triazolo[1,5-a]pyrimidine-6carboxylate 2 as a single product, as evident by TLC and IR spectra by the appearance of two $\mathrm{CO}$ group. Compound $\mathbf{2}$ gave hydroxamic acid $\mathbf{3}$ when reacted with hydroxylamine hydrochloride. Reaction of compound 2 with hydrazine hydrate in methanol afforded the corresponding hydrazide 4. Condensation of hydrazide 4 with aromatic aldehydes led to corresponding Schiff bases 5 a-g. The structures were confirmed on the basic of elemental analyses, spectral data. ${ }^{1} \mathrm{H}-\mathrm{NMR}$ showed downfield signals at $\delta$ 10.72-12.9 (CONH), 7.95-7.58 $(\mathrm{N}=\mathrm{CH})$ and disappearance of signals of $\mathrm{NH}_{2}$ in addition of aromatic protons which appeared at the expected chemical shifts. Refluxing of hydrazide 4 with appropriate isothiocyanate gave thiosemicarbazides 6a-f. The structures were confirmed on the 
basic of elemental analyses, spectral data. ${ }^{1} \mathrm{H}-\mathrm{NMR}$ showed signals at $\delta$ 10.9-10.5 (CONH), 9.3-9.78 (NHCS), and 4.00-8.74 $\left(\mathrm{N}^{4}-\mathrm{H}\right)$. All other aromatic and aliphatic protons were observed at expected regions. Physical data of the synthesized compounds are shown in Table 1.

\section{Antimycobacterial activity}

The synthesized compounds $\mathbf{3 , 4 , 5}(\mathbf{a}-\mathbf{g})$ and $\mathbf{6}(\mathbf{a}-\mathbf{f})$ were tested for their primary antimycobacterial activity against Mycobacteria tuberculosis $\mathrm{H}_{37} \mathrm{Rv}$, IC90 and IC50 were determined as shown in Table 2.

Compound $\mathbf{5 e}$ and $\mathbf{5 b}$ showed activity with IC90 (6.672, 7.362 $\mu \mathrm{g} / \mathrm{mL}$ respectively) and IC50 (4.627, $6.382 \mu \mathrm{g} / \mathrm{mL}$ respectively) while compounds 5c and 5d showed weak activity with IC90>100 and IC50 (82.237, 29.67). The remaining compounds showed no activity. From the above results we can conclude that Schiff bases as a unit appears to be a useful scaffold for the antimycobacterial agents.<smiles>CCOC(=O)C(OCC)=C(OCC)C(=O)OCC</smiles>

1<smiles></smiles>

4<smiles>C#CCCCCCC(=O)c1c[nH]c2nc(C(F)(F)F)nn2c1=O</smiles>

$5 a-g$<smiles>O=C(NNC1CCCN1)c1c[nH]c2nc(C(F)(F)F)nn2c1=O</smiles>

$6 a-f$

$\mathrm{Ar}=\mathrm{ph}, p-\mathrm{OCH}_{3}-\mathrm{ph}, 3,5-\left(\mathrm{OCH}_{3}\right)_{2}-\mathrm{ph}, 4-\mathrm{OH}, 3-\mathrm{OCH}_{3}-\mathrm{ph}, p-\mathrm{N}\left(\mathrm{CH}_{3}\right)_{2}-\mathrm{ph}, p-\mathrm{Cl}-\mathrm{ph}, 3-$ pyridyl

$\mathrm{R}^{1}=\mathrm{CH}_{3}, \mathrm{C}_{2} \mathrm{H}_{5}, \mathrm{CH}_{2} \mathrm{CH}=\mathrm{CH}_{2}, \mathrm{Ph}, p-\mathrm{CH}_{3}-\mathrm{Ph}$, c.hexyl

Scheme 1: Synthesis of the target compounds. 
Table 2: Antitubercular in vitro activity of the test compounds expressed as IC90, IC50 of mycobacterium tuberculosis $\mathrm{H}_{37} \mathrm{Rv}$.

\begin{tabular}{|c|c|c|c|c|c|}
\hline $\begin{array}{c}\text { Compd. } \\
\text { No. }\end{array}$ & Sample ID & Assay & IC90 & IC50 & Activity \\
\hline $\mathbf{3}$ & 408898 & MABA & $>100$ & $>100$ & Inactive \\
\hline $\mathbf{4}$ & 408897 & MABA & $>100$ & $>100$ & Inactive \\
\hline $\mathbf{5 a}$ & 408905 & MABA & $>100$ & $>100$ & Inactive \\
\hline $\mathbf{5 b}$ & 408907 & MABA & 7.362 & 6.382 & Active \\
\hline $\mathbf{5 c}$ & 408908 & MABA & $>100$ & 82.237 & Weakly active \\
\hline $\mathbf{5 d}$ & 408909 & MABA & $>100$ & 29.670 & Weakly active \\
\hline $\mathbf{5 e}$ & 408906 & MABA & 6.672 & 4.627 & Active \\
\hline $\mathbf{5 f}$ & 408910 & MABA & $>100$ & $>100$ & Inactive \\
\hline $\mathbf{5 g}$ & 408911 & MABA & $>100$ & $>100$ & Inactive \\
\hline $\mathbf{6 a}$ & 408899 & MABA & $>100$ & $>100$ & Inactive \\
\hline $\mathbf{6 b}$ & 408900 & MABA & $>100$ & $>100$ & Inactive \\
\hline $\mathbf{6 c}$ & 408901 & MABA & $>100$ & $>100$ & Inactive \\
\hline $\mathbf{6 d}$ & 408902 & MABA & $>100$ & $>100$ & Inactive \\
\hline $\mathbf{6 e}$ & 408903 & MABA & $>100$ & $>100$ & Inactive \\
\hline $\mathbf{6 f}$ & 408904 & MABA & $>100$ & $>100$ & Inactive \\
\hline
\end{tabular}

Conc $\mu \mathrm{g} / \mathrm{mL}$

IC50, IC90: is the concentration where a drug inhibits the TB strain by $50 \%$ or 90\%. Compounds are considered active if IC90 $\leq 10 \mu \mathrm{g} / \mathrm{mL}$.

\section{Antimicrobial activity}

The synthesized compounds were tested for their in-vitro antimicrobial activity against Escherichia coli (E. coli, ATCC 25922), Serratia marcescens, DMS 1608, Pseudomonas aeruginosa as gram negative bacteria. Bacillus cereus, and Staphylococcus aureus ATCC 25923 as Gram positive bacteria. Antifungal activity against Aspergillus niger, Aspergillus flavus, Candida albicans, (WT-S) Scopulariopsis brevicaulis, Fusarium oxysporum and Geotrichum candidum by the method described by Kwon-Chung and Bennett ${ }^{17}$. The tesed compounds were allowed to diffuse readily from wells into the medium inoculated with microorganism. Ampicillin trihydrate, Nalidixic acid and Clotrimazole were used as standard antibacterial and antifungal agents, respectively. As shown in Table 3. Some of the compounds tested were illustrated significant antibacterial and antifungal activity when compared with reference drugs. 
Table 3: Antimicrobial activity of the synthesized compounds.

\begin{tabular}{|c|c|c|c|c|c|c|c|c|c|c|c|}
\hline \multicolumn{12}{|c|}{ In vitro activity-zone of inhibition in $\mathrm{mm}(\mathrm{MIC}$ in $\mu \mathrm{g} / \mathrm{ml})$} \\
\hline No. & $\begin{array}{c}E . \\
\text { Coli } \\
\end{array}$ & $\begin{array}{c}S . \\
\text { marcescens }\end{array}$ & B. cereus & $\begin{array}{c}P . \\
\text { aeruginosa }\end{array}$ & $\begin{array}{c}\text { Staf. } \\
\text { Aureus }\end{array}$ & $\begin{array}{c}\text { Asp } \\
\text { niger }\end{array}$ & $\begin{array}{c}\text { Asp. } \\
\text { flavus }\end{array}$ & $\begin{array}{c}\text { Can. } \\
\text { albicans }\end{array}$ & $\begin{array}{c}\text { Scop. } \\
\text { brevicaulis }\end{array}$ & $\begin{array}{c}\text { Fus. } \\
\text { Oxysporum }\end{array}$ & $\begin{array}{c}\text { Geo. } \\
\text { candidum }\end{array}$ \\
\hline 3 & $12(75)$ & $12(125)$ & $16(250)$ & $30(1000)$ & $10(250)$ & $16(250)$ & $10(25)$ & $12(500)$ & $10(250)$ & - & $16(125)$ \\
\hline 5a & $16(25)$ & $16(25)$ & $16(50)$ & $8(125)$ & $12(500)$ & $14(250)$ & $10(250)$ & $8(500)$ & $18(250)$ & $22(500)$ & $14(75)$ \\
\hline $\mathbf{5 b}$ & $8(250)$ & $8(500)$ & $8(250)$ & $8(250)$ & - & - & - & - & - & . & - \\
\hline $5 c$ & $8(50)$ & $10(500)$ & $10(250)$ & $10(250)$ & - & - & - & - & - & - & - \\
\hline 5d & - & $20(125)$ & - & - & $12(125)$ & - & - & $12(50)$ & $14(250)$ & - & $10(50)$ \\
\hline $5 e$ & - & - & - & $\begin{array}{ll}- & - \\
\end{array}$ & $\begin{array}{ll}- & - \\
\end{array}$ & & & & & & \\
\hline 5f & $12(500)$ & $16(500)$ & $17(500)$ & 19(500) & $14(500)$ & - & - & - & $10(500)$ & - & - \\
\hline $5 \mathrm{~g}$ & 11(50) & 10 (125) & - & - & - & & & & & & \\
\hline $6 b$ & $10(75)$ & $12(125)$ & $12(75)$ & $8(250)$ & - & $12(500)$ & $14(500)$ & $12(75)$ & - & $8(500)$ & $8(250)$ \\
\hline $6 c$ & - & - & - & - & - & & & & & & \\
\hline 6d & - & - & - & - & - & - & - & - & - & - & - \\
\hline $6 e$ & & & & & & - & - & - & - & - & - \\
\hline Nali & $14(12.5)$ & $20(12.5)$ & $20(25)$ & - & $26(25)$ & & & & & & \\
\hline Ampi & $12(500)$ & - & $8(250)$ & - & $12(500)$ & & & & & & \\
\hline Clotri & & & & & & $22(25)$ & $21(25)$ & $20(25)$ & $26(25)$ & $22(25)$ & $21(25)$ \\
\hline
\end{tabular}


The MIC values were generally within the range of $(25-500 \mu \mathrm{g} / \mathrm{mL})$ against all evaluated strains. In comparing their MIC values, compounds 3, 5a, 6b showed higher activity than ampicillin against almost strains, and activity range from half to sixth activity of nalidixic acid against E. coli. Compounds $\mathbf{5 b}, \mathbf{5 c}, \mathbf{5 d}, \mathbf{5 f}, \mathbf{5 g}$ showed higher activity or similar activity as ampicillin against certain strains as shown in Table 2 . Compounds 3, 5a, 5b, 5c, 5f, 6b showed activity against $P$. aeruginosa, while nalidixic acid possessed no activity. Compounds $\mathbf{3}$, 5a, 5d, 6b possess antifungal activity with MIC values range from (75-500 $\mu \mathrm{g} / \mathrm{mL}$ ) which is comparable to or less than clotrimazole against $A$. flavus, G. candidum and C. albicans.

\section{Conclusion}

It was found that the most active derivative of the tested compounds against certain strains of bacteria and fungi was 2-(trifluoromethyl)-4,7dihydro-7-oxo-1,2,4-triazolo[1,5-a] pyrimidine-6-hydroxamic acid $\mathbf{3}$. The Schiff bases bearing electron donating groups in $p$-position of the phenyl ring possessed good antimycobacterial activity (5e and 5b). The unsubstituted Schiff base, N-Benzylidine-2-(trifluoromethyl)4,7-dihydro-7-oxo-1,2,4-triazolo[1,5a] pyrimidine-6-carbohydrazide $\mathbf{5 a}$ was found to be the most active member of series 5, substitution on the phenyl ring decrease activity. In thio-semicarbazide series $\mathbf{6}$, it was found that compound $\mathbf{6 b}$ bearing ethyl group at $\mathrm{N}^{4}$ of thiosemicarbazide was the only active compound against bacteria and fungi.

\section{Acknowledgment}

Antimycobacterial data were provided by the Tuberculosis Antimicrobial Acquisition and Coordinating Facility (TAACF) through a research and development contract with the U.S. National Institute of Allergy and Infectious Diseases.

Antimicrobial activity were done in Mycological Center, Assiut University, Assiut, Egypt.

\section{REFERENCES}

1- Y. -L. Chen, K. -C. Fang, J. -Y. Sheu, S. -L. Hsu, and C. -C. Tzeng, J. Med. Chem., 44, 2374 (2001).

2- A. M. Abdelal, M. M. Gineinah and S. M. Kheira, Med. Chem. Res., 9, 277 (1999).

3- G. Anquetin, M. Rouquayrol, N. Mahmoudi, M. Santillana-Hayat, R.Gozalbes, J. Greiner, K. Farhati, F. Derouin, R. Guedj and P. Vierling, J. Bioorg. Med. Chem. Lett., 14, 2773 (2004).

4- Anand V. Shindikar and C. L. Viswanathan, J. Bioorg. Med. Chem. Lett., 15, 1803 (2005).

5- C. Boyer-Joubert, E. Lorthiois and F. Moreau, in "Annual Reports in Medicinal Chemistry", Volume 38 , "Elsevier Academic Press", Amsterdam, 2003, p. 347.

6- J. Matsumoto and S. Minami, J. Med Chem., 18, 74 (1975). 
7- P. M. Gilis, A. Haemers and W. Bollaert, J. Heterocyclic Chem., 17, 717 (1980).

8- N. Ito and S. Yamamura, Japanese Patent 7129,870 (1971).

9- S. M. Bayomi, K. E. Price and J. W. Sr. Sowell, J. Heterocyclic Chem., 22, 83 (1985).

10- S. M. Bayomi, K. E. Price and J. W. Sr. Sowell, ibid., 22, 729 (1985).

11 - N. J. Hares, A. M. Al Obaid, S. M. Bayomi and M. M. El-Enany, Egypt. J. Pharm. Sci., 32, 303 (1991).

12- T. Yoshida, Y. Yamamoto, H. Orita, M. Kakiuchi, Y. Takahashi, M. Itakura, N. Kado, S. Yasuda, H. Kato and Y. Itoh, Chem. Pharm. Bull., 44, 1376 (1996).
13- V. V. Lipson, I. V. Ignatenko, S. M. Desenko, S. V. Shishkina, O. V. Shinhkin, S. A. Komykhov, N. V. Logvinenko, V. D. Orlov and H. Meier, J. Heterocyclic Chem., 40, 1081 (2003).

14- H. A. Al-Kamees, O. A. Al-Deeb and S. M. Bayomi, Indian J. Heterocyclic Chem., 2, 237 (1993).

15- V. A. Loprev and T. N. Rakhmatulina, Zh. Obshch. Khim, 53, 1684 (1983), (Through Chem. Abst., 99, 139865, 1983).

16- L. Collins and S.G. Franzblau, Antimicrob. Agents Chemother., 41, 1004 (1997).

17- K. J. Kwon-Chung and J. E. Bennet, Medicinal Mycology Lea and Febiger, Philadelphia, 1992, p. 866 